\title{
gु
}

\section{Competition between Polar and Nonpolar Lattice Distortions in Oxide Quantum Wells: New Critical Thickness at Polar Interfaces}

\author{
J. Gazquez, ${ }^{1}$ M. Stengel,,${ }^{1,2}$ R. Mishra, ${ }^{3}$ M. Scigaj, ${ }^{1}$ M. Varela, ${ }^{4,5}$ M. A. Roldan, ${ }^{5}$ \\ J. Fontcuberta, ${ }^{1}$ F. Sánchez, ${ }^{1}$ and G. Herranz ${ }^{1}$ \\ ${ }^{1}$ Institut de Ciència de Materials de Barcelona, Campus de la UAB, 08193 Bellaterra, Spain \\ ${ }^{2}$ ICREA, Pg. Lluís Companys 23, 08010 Barcelona, Spain \\ ${ }^{3}$ Department of Mechanical Engineering and Materials Science, Washington University in St. Louis, St. Louis, Missouri 63130, USA \\ ${ }^{4}$ Materials Science and Technology Division, Oak Ridge National Laboratory, Tennessee 37831-6071, USA \\ ${ }^{5}$ Departamento de Física de Materiales and Instituto Pluridisciplinar, Universidad Complutense de Madrid, Madrid 28040, Spain
}

(Received 16 March 2017; revised manuscript received 12 June 2017; published 7 September 2017)

Two basic lattice distortions permeate the structural phase diagram of oxide perovskites: antiferrodistortive (AFD) rotations and tilts of the oxygen octahedral network and polar ferroelectric modes. With some notable exceptions, these two order parameters rarely coexist in a bulk crystal, and understanding their competition is a lively area of active research. Here we demonstrate, by using the $\mathrm{LaAlO}_{3} / \mathrm{SrTiO}_{3}$ system as a test case, that quantum confinement can be a viable tool to shift the balance between AFD and polar modes and selectively stabilize one of the two phases. By combining scanning transmission electron microscopy (STEM) and first-principles-based models, we find a crossover between a bulklike $\mathrm{LaAlO}_{3}$ structure where AFD rotations prevail, to a strongly polar state with no AFD tilts at a thickness of approximately three unit cells; therefore, in addition to the celebrated electronic reconstruction, our work unveils a second critical thickness, related not to the electronic properties but to the structural ones. We discuss the implications of these findings, both for the specifics of the $\mathrm{LaAlO}_{3} / \mathrm{SrTiO}_{3}$ system and for the general quest towards nanoscale control of material properties.

DOI: 10.1103/PhysRevLett.119.106102

Polar lattice distortions [1,2] and "antiferrodistortive" (AFD) tilts of the $\mathrm{BO}_{6}$ octahedral network [3,4] are ubiquitous in the physics of $A B \mathrm{O}_{3}$ perovskite oxides and largely responsible for their interesting functional properties. Indeed, the former potentially leads to ferroelectricity, while the latter often has a dramatic impact over the orbital and magnetic orders. Although some notable exceptions exist (e.g., $\mathrm{BiFeO}_{3}$ ), the two order parameters tend to be mutually contraindicated in a bulk perovskite crystal [3]. Such a competition has often been used in the continuing search for new multiferroics or, more generally, to engineer exotic phases via atomic-scale design, as in the recent report of polar metals at perovskite interfaces [5]. Overall, controlling the balance between the competing polar and AFD phases offers exciting opportunities to realize novel properties "à la carte." Such a balance is often delicate, as both types of lattice distortions and their mutual couplings are governed by a subtle interplay of short-range covalency (related to $B-\mathrm{O}$ bonding) and long-range electrostatic (or elastic) effects [6].

Atomic-scale confinement appears as an ideal tool to tune the competition between different order parameters, as it is known to profoundly alter the spectrum of lattice and electronic excitations of crystalline materials [7-11]. In ferroelectrics, for example, size effects play a dramatic role in determining the stability of the polar state $[12,13]$. The driving force that determines the critical thickness for ferroelectricity is of an electrostatic nature and manifests itself as a "depolarizing" electric field that is antiparallel to the film's polarization. Therefore, the question of whether nonferroelectric (and hence insensitive to macroscopic depolarizing effects) modes such as the AFD tilts may also experience a "critical thickness" becomes particularly interesting one. Apart from the academic interest, suppressing (or partially destabilizing) the AFD tilts may be also useful for practical applications. Manganites and nickelates are good examples where magnetic and transport properties are sensitive to AFD tilts [14].

The interface between $\mathrm{LaAlO}_{3}$ and $\mathrm{SrTiO}_{3}$ - two prominent compounds in the field of oxide electronics [15] provides an excellent model system to address the effect of confinement on AFD and/or polar lattice distortions. According to the "polar catastrophe model," the electrostatic mismatch at the $\mathrm{LaAlO}_{3} / \mathrm{SrTiO}_{3}$ interface produces a strong internal electric field in the $\mathrm{LaAlO}_{3}$ layer at subthreshold thicknesses $(<4$ u.c.), which is neutralized at larger thicknesses via the spontaneous formation of a high-mobility 2DEG [16-19]. The subthreshold electric field has been predicted $[20,21]$ to strongly polarize the $\mathrm{LaAlO}_{3}$ lattice, but a direct experimental confirmation is still missing. Besides, there is a structural mismatch at the interface as well: $\mathrm{LaAlO}_{3}$, either in bulk or thin-film form, presents large AFD tilts at room temperature, while the $\mathrm{SrTiO}_{3}$ substrate adopts an undistorted cubic phase, which disturbs the continuity of the $\mathrm{O}$ network. Therefore, in ultrathin $\mathrm{LaAlO}_{3} / \mathrm{SrTiO}_{3}$ heterostructures, we have the unique 
coexistence of an electrostatic and a structural discontinuities. As both confinement and electric fields can be controlled experimentally, they could be used as a switch to toggle the ground state of the system between competing phases, see Fig. 1(a).

Here we used high-resolution annular bright-field (ABF) in STEM to map the positions of all ions (including $\mathrm{O}$ columns) across the $\mathrm{LaAlO}_{3} / \mathrm{SrTiO}_{3}$ interface, for a varying thickness $t$ of the $\mathrm{LaAlO}_{3}$ overlayer. We find that, for a subcritical $(t<4$ u.c. $) \mathrm{LaAlO}_{3}$ film, nonpolar AFD distortions are suppressed in favor of a strong polar distortion within the film. This result provides unambiguous evidence in support of the "polar-catastrophe" model and a direct demonstration of how the AFD modes can be controlled at the nanoscale. A first-principles based numerical model indicates that the spatial confinement within $\mathrm{LaAlO}_{3}$ is indeed the driving force for the AFD suppression. It also predicts a non-negligible interaction (via a repulsive (a) Polar distortions

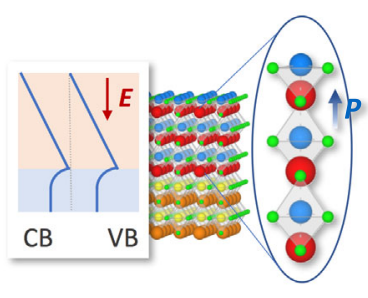

Confined $\mathrm{LaAlO}_{3}$ lattice

(b)

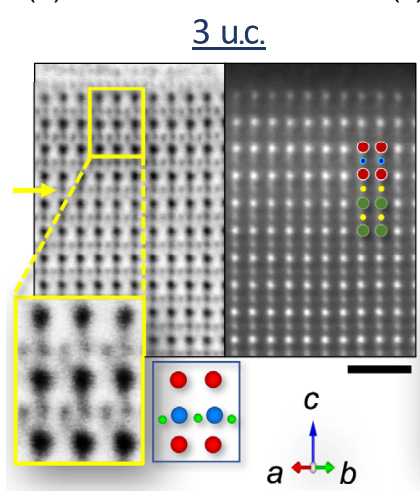

Nonpolar distortions

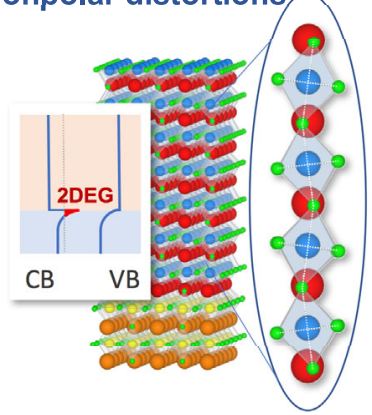

Extended $\mathrm{LaAlO}_{3}$ lattice
FIG. 1. (a) Schematic structures of the polar displacements and octahedral tilts in $\mathrm{LaAlO}_{3}$ film of thicknesses below and above the critical one for $2 \mathrm{DEG}$ formation in $\mathrm{SrTiO}_{3}$ in $\mathrm{LaAlO}_{3} / \mathrm{SrTiO}_{3}$ heterostructures: $<4$ u.c. (left panel) and $>4$ u.c. (right panel), respectively. (b),(c) Atomic-scale mapping of the interface. Upper panel, (b),(c) $Z$ contrast (right) and ABF (left) simultaneously acquired STEM images of 3- and 7-u.c.-thick $\mathrm{LaAlO}_{3} / \mathrm{SrTiO}_{3}$ samples, respectively, viewed along the [110] zone axis. The $[110]_{\mathrm{pc}}$ projection allows visualizing the $\mathrm{O}$ sublattice buckling. Lower panel, zoomed out regions of the $\mathrm{ABF}$ images of the 3- and 7-u.c.-thick $\mathrm{LaAlO}_{3}$ layers along with schematics of the $\mathrm{LaAlO}_{3}$ structure showing the revealed distortions. Scale bar, $1 \mathrm{~nm}$. biquadratic term) between the polar modes and the AFD tilts, which is important for achieving a quantitative agreement with the experimentally observed critical thickness. Our work reveals two simultaneous phase transitions in a 3 u.c. $\mathrm{LaAlO}_{3}$ : a metal-insulator transition at the interface driven by the 2DEG formation and another transition involving lattice deformations in which AFD modes disappear and a polar state emerges in their place.

Figures 1(b) and 1(c) show the Z-contrast (upper right panels) and $\mathrm{ABF}$ (upper left panels) images of 3- and 7u.c.-thick $\mathrm{LaAlO}_{3}$ layers, respectively. Images of a 5-u.c. -thick sample can be found in Supplemental Material [22]. The ABF images reveal important variations of the $\mathrm{LaAlO}_{3}$ structure as a function of its thickness. These structural changes were quantified using a center-of-mass refinement on the contrast-inverted $\mathrm{ABF}$ images to determine the atomic position. We verified that any bias introduced by the varying background signal close to the interface is negligible; see Supplemental Material [22]. We accurately determined the location of all the atomic sites along with the interface plane and obtained a detailed real-space map of both polar and AFD modes within the $\mathrm{LaAlO}_{3}$ film.

First, we extracted [Figs. 2(a)-2(d)] the polar shifts $\left(\delta_{B}\right.$, $\delta_{\mathrm{O}}$ ), whereby the $B$ and $\mathrm{O}$ sublattices are off-centered with respect to the $A$ sublattice. In the 3-u.c.-thick $\mathrm{LaAlO}_{3}$ layer, both $\delta_{B}$ and $\delta_{\mathrm{O}}$ are negative; i.e., the $\mathrm{Al}$ and $\mathrm{O}$ atoms move towards the interface relative to the La sublattice. The fact that $\mathrm{Al}$ atoms also move downward may seem counterintuitive; however, as our model calculations predict-see Fig. S10 - in the presence of an electric field the polar displacements are dominated by the upward movement of the La. Moreover, the measured $\mathrm{Al}$ and $\mathrm{O}$ shifts show excellent agreement with our first-principles calculations, and the corresponding nonzero relative displacement $\left(\delta_{B}>\delta_{\mathrm{O}}\right)$ indicates a net electrical dipole pointing away from the substrate [Fig. 2(c)]. This finding confirms the predicted polar distortions in $\mathrm{LaAlO}_{3}$ [20] and is consistent with the presence of an internal field in subcritical $\mathrm{LaAlO}_{3}$ layers. To verify this point, we performed first-principles calculations of $\mathrm{LaAlO}_{3}$ under the influence of a macroscopic electric displacement $D_{z}$. After structural relaxation, we extracted $\delta_{B}$ and $\delta_{\mathrm{O}}$ as a function of $D_{z}$ (Fig. S10). Remarkably, we find excellent agreement with the experimentally measured values for $D_{z} \sim 0.65 \times\left(e / 2 a_{0}^{2}\right) \quad\left(a_{0}\right.$ is the in-plane lattice parameter), confirming the electrostatic nature of the observed distortions. The resulting internal field, although smaller than the expected value $\left(D_{z}=\left(e / 2 a_{0}^{2}\right)\right)$ for the ideal insulating interface $[17,18]$, provides unequivocal support to the so-called "polar catastrophe" model [16]. Indeed, our measured polar shifts in the 5-u.c. (see Supplemental Material [22]) and 7-u.c. samples gradually decrease [Figs. 2(d) and S3], as anticipated for above-threshold (and hence electrostatically compensated) films ( $>4$ u.c.). Interestingly, polar distortions in $\mathrm{SrTiO}_{3}$ near the interface are also visible, in agreement with first-principles predictions [21] and earlier experimental observations [33,34]. Besides, 


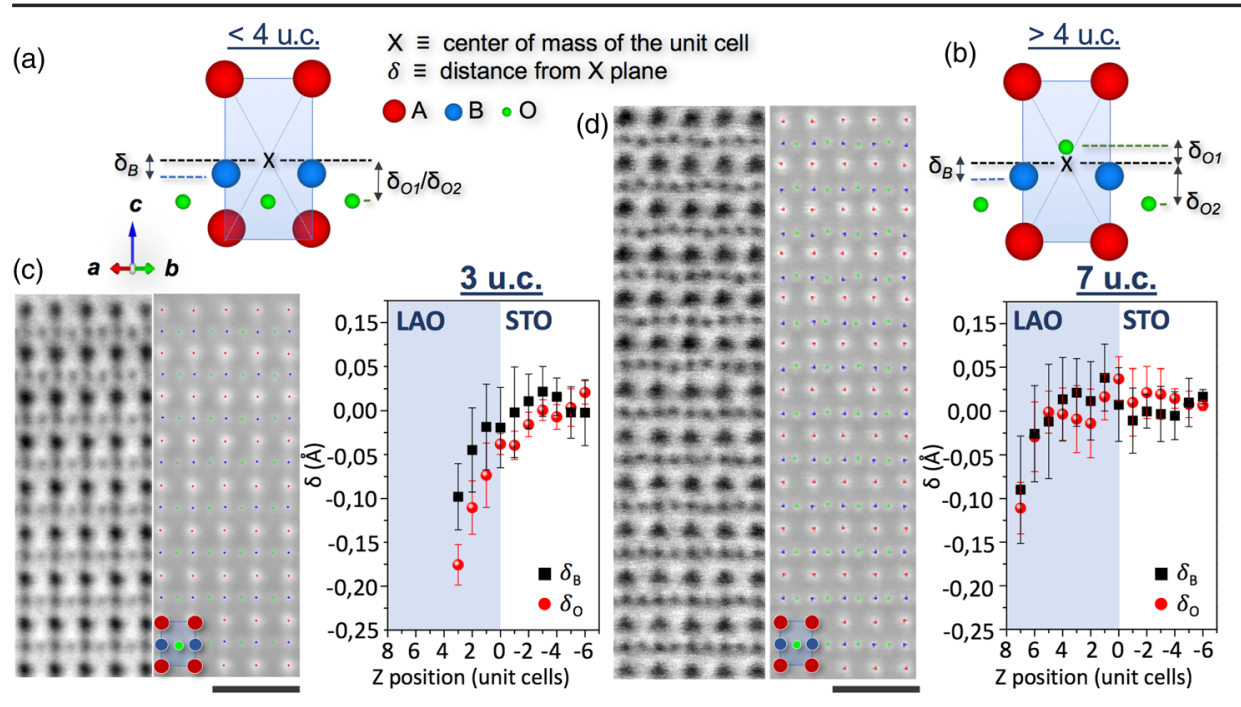

FIG. 2. (a),(b) Schematics of the distortions revealed by the ABF images shown in Fig. 1. The perovskite $\mathrm{ABO}_{3}$ unit cells viewed along the [110] zone axis show the distance $\delta$ from the center of mass of the unit cell (marked with a cross) and that of the $B$ and $\mathrm{O} 1$ and $\mathrm{O} 2$ sites for $<4$ u.c. (a) and $>4$ u.c. (b) thick $\mathrm{LaAlO}_{3}$ layers. The schemes show the displacements (not in scale) of $B$ and $\mathrm{O}$ atoms from the center of the unit cell. From left to right, (c) and (d) show $\mathrm{ABF}$ and inverted $\mathrm{ABF}$ images along with the averaged values of the distance $\delta$ of the $B$ (black squares) and $\mathrm{O}$ (red circles) sites from the center of mass of each unit cell (right panel) as a function of position of the 3- and 7-u.c.-thick samples, respectively. Scale bar, $1 \mathrm{~nm}$.

our electron energy loss spectroscopy (EELS) analysis shows that the structural distortions penetrates further into the $\mathrm{SrTiO}_{3}$ substrate than the ionic interdiffusion length, which rules out the hypothesis of the former originating from interface chemistry effects, see [22].

Second, we isolated the tilting patterns of the $\mathrm{BO}_{6}$ octahedral units by measuring the rippling within the visible portion of the $\mathrm{O}$ sublattice, see Fig. 3(a). Such rippling is due to the presence of octahedral tilts along the $[100]_{\mathrm{pc}}$ and $[010]_{\mathrm{pc}}$ directions corresponding to the amplitude of the $[110]_{\mathrm{pc}}$-oriented AFD mode $\left(a^{-} a^{-} a^{0}\right.$ pattern), which we quantify via the projected rotation angle $\alpha$. In both the 5- and the 7-u.c.-thick layers, the rotation angles are overall quite large near the film surface $\left[\alpha \approx 3.5^{\circ}-4^{\circ}\right.$, Fig. 3(b)] and progressively decrease to zero when moving towards the $\mathrm{SrTiO}_{3}$ substrate. Both the measured in-plane tilt axis and distortion amplitude are consistent with the first-principles predictions of Hatt and Spaldin for tensilestrained $\mathrm{LaAlO}_{3}$ films [35]; also, a decrease of $\alpha$ towards the interface was expected-recall that $\mathrm{SrTiO}_{3}$ at room temperature has a cubic structure with no AFD tilts. In contrast, in the 3-u.c.-thick layer [Fig. 3(b)], the rotations are completely suppressed, except for the uppermost Al-O-Al plane, where $\alpha \approx 1.2^{\circ}$. Remarkably, such an abrupt suppression of the oxygen AFD tilts occurs at the same critical thickness at which we observe a drastic increase in the polar distortions [see Fig. 2(c)].

To shed some light on the observed evolution of the polar and antiferrodistortive degrees of freedom as a function of thickness, we considered a Landau-type energy functional that describes the energetics of the AFD modes and their interaction with electric fields:

$$
F\left(\phi, D_{z}\right)=\frac{A}{2} \phi^{2}+\frac{B}{4} \phi^{4}+\frac{C}{2} \phi^{2} D_{z}^{2}+\frac{G}{2}\left(\frac{\partial \phi}{\partial z}\right)^{2} .
$$

Here $\phi$ is related to the amplitude of the in-plane AFD tilt (the rotation angle $\alpha$ ). The first two terms in Eq. (1) encode the tendency of the crystal to distort spontaneously, where the negative $A$ and the positive quadratic coefficient $B$ guarantees the overall thermodynamic stability. The third term in Eq. (1) describes the biquadratic repulsion between the AFD tilts and the internal electric field (described by the displacement $D_{z}$ ), well known in ferroelectric perovskites [36-38]. $C$ is an "effective" biquadratic coefficient that implicitly includes the contribution of secondary lattice modes [39]. The fourth term in Eq. (1) penalizes AFD mode gradients, which appear inevitably because at room temperature the AFD rotations must vanish at the boundary with $\mathrm{SrTiO}_{3}$. Except for the gradient couplings, this model (and the computational approach to calculating the coefficients) is similar in spirit to that of Ref. [40].

The coefficients $A, B, C$, and $G$ in Eq. (1) were extracted from a set of first-principles calculations, which we performed in the framework of the local density approximation to the Density Functional Theory (see Supplemental Material [22]). In particular, $A$ and $B$ were obtained by computing the energy difference between the undistorted lattice and the fully relaxed structure with an optimized $a^{-} a^{-} a^{0}$ pattern; $C$ was extracted from two sets of constrained- $D$ calculations, performed either with or without relaxing the AFD tilts. An additional quadratic-quartic $\phi^{2} D_{z}^{4}$ term was introduced in Eq. (1) to improve the accuracy of the fitting at large fields [see Fig. 3(c)]. The coefficient $G$ was obtained by fitting the lowest unstable phonon branch of the undistorted cell along the RM path in reciprocal space [i.e., the $\phi_{x}, \phi_{y}$ doublet corresponding to in-plane AFD tilts indicated by the dashed red curve in Fig. 3(d)]. Interestingly, above a critical wave vector $\left(q_{\text {crit }}=0.16\right)$, the $\phi_{x}, \phi_{y}$ phonon frequency becomes positive, which implies that at wavelengths shorter than $\left(1 / q_{\text {crit }}\right) \sim 6.5$ u.c. a spatially modulated AFD distortion cannot occur spontaneously. This 
(a)
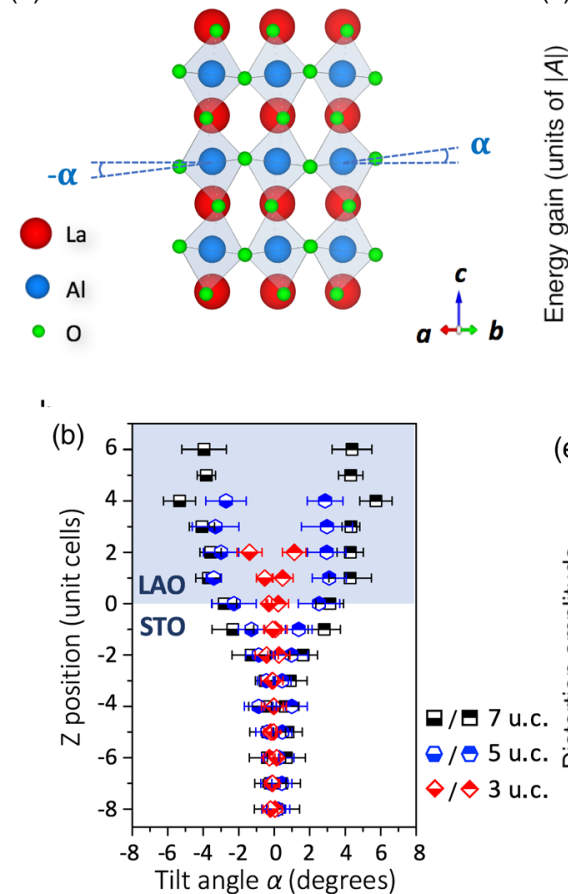

e)
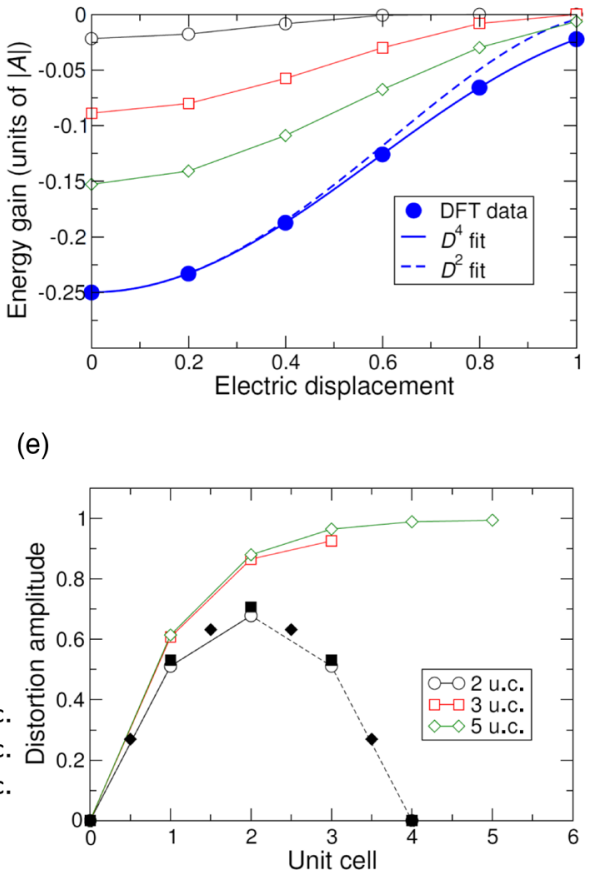

(d)

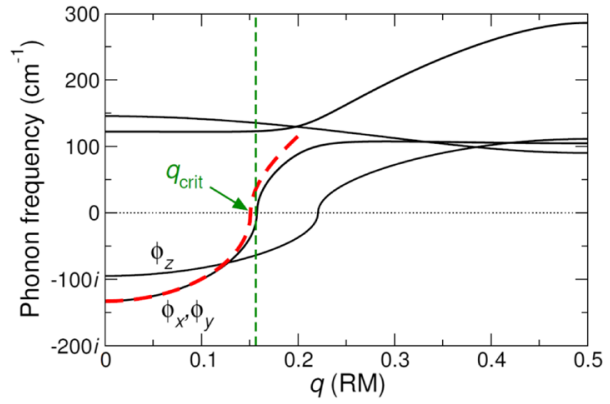

(f)

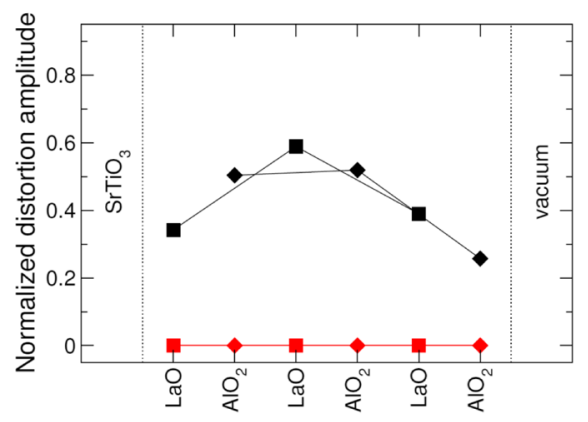

FIG. 3. (a) [110] view of the $\mathrm{LaAlO}_{3}$ structure showing octahedral tilts. (b) $B-\mathrm{O}-B$ tilt angle along the $z$ axis, as a function of thickness. The tilting of the 3-, 5-, and 7-u.c.-thick samples are shown in red, blue, and black, respectively. The error bars show the standard deviation with respect to the average for each atomic layer. (c) Energy per unit cell associated to the tilts as a function of the dielectric displacement $D_{z}$. The solid blue circles show the first-principles results for a uniform tilt pattern, calculated as the energy difference between a distorted and undistorted geometry. The solid and dashed blue curves are fits, respectively including or excluding the additional $\phi^{2} D_{z}^{4}$ term. The empty symbols are model results for confined tilt patterns: Results for 2-u.c. (black circles), 3-u.c. (red squares), and 5-u.c. (green diamonds) films are shown. The electric displacement $D_{z}$ is in units of $e / 2 a_{0}^{2}$. (d) Phonon dispersion curves of the reference undistorted (but elastically strained) cell along the RM segment in the Brillouin zone. The dashed red curve shows a quadratic fit of the lowest unstable branch. (e) Distortion amplitude as a function of film thickness [same color and symbol code as in (b)], as predicted by the model Eq. (1). The 2-u.c. film with asymmetric boundary conditions (solid line) is shown as an equivalent 5-u.c. segment with symmetrical blocking boundary conditions (dashed line). The solid symbols correspond to the amplitudes extracted from an explicit first-principles calculation (see Supplemental Material [22]). (f) AFD distortions (black symbols) in a thin (3-u.c.-thick) $\mathrm{LaAlO}_{3}$ layer deposited on $\mathrm{SrTiO}_{3}$ substrate, with the internal field neutralized via external surface charges (see Supplemental Material [22]). In the "pristine" system (red symbols), the tilts as they are suppressed by the strong internal field.

observation embodies the essence of the confinement effects that we discuss in this work: An unstable lattice mode is eventually suppressed if the positive gradient energy becomes too strong.

After setting the boundary conditions that best reproduce the experimental observations [Fig. 3(b) shows that the tilt amplitude in $\mathrm{LaAlO}_{3}$ behaves qualitatively as air vibrations in an open-ended pipe, i.e., with a node at the interface and a maximum at the free surface], we initially solved our model in the absence of an electric field. We obtain a critical thickness for the emergence of AFD tilts of about 2 u.c. [Fig. 3(e)], which is significantly smaller than the experimental one. The key to solving this inconsistency resides in the effect of internal fields on the AFD modes. To demonstrate this important point, we used Eq. (1) to estimate the field-induced change in the energy gain $\left(\Delta E_{\mathrm{AFD}}\right)$ associated to the relaxation and, therefore, the presence of the AFD tilts. The results [Fig. 3(c)] show a clear general trend; namely, internal fields significantly increase the critical thickness for the appearance of AFD tilts. For instance, distortions in the 2-u.c. system are suppressed when $D_{z}>0.6 \times\left(e / 2 a_{0}^{2}\right)$. For the 3-u.c. layer, a larger field $D_{z} \sim\left(e / 2 a_{0}^{2}\right)$ is required [Fig. 3(c)], roughly the expected value for the ideal electronic reconstruction $[17,18]$. Therefore, both internal fields and spatial confinement are necessary to explain the unexpected suppression of the tilts in the 3 -u.c. $\mathrm{LaAlO}_{3}$ film. This result and the direct observation of strong polar distortions provide a strong case supporting the polar catastrophe model, which predicts the existence of such a field for insulating interfaces ( $<4$ u.c.).

In summary, our work reveals that the strong competition between octahedral tilts and polar displacements results in the emergence of two simultaneous reconstructions in the $\mathrm{LaAlO}_{3} / \mathrm{SrTiO}_{3}$ system [Fig. 1(a)]. In addition to the celebrated metal-insulator transition, our results show that an extra reconstruction occurs on the $\mathrm{LaAlO}_{3}$ side, driven by the action of an electric field on AFD modes. This indicates that controlling electric fields inside a lattice may 
provide an additional way to reversibly activate octahedral distortions in atomically confined perovskites, possibly enabling dynamic modulation of their functionalities. We explored this possibility with an additional computational experiment in which we artificially neutralized the internal field within the $\mathrm{LaAlO}_{3}$ film (Supplemental Material [22]). The results [Fig. 3(f)] show that the suppression of the internal field triggers the reemergence of the AFD distortions, consistent with the predictions of our continuum model. This test opens up enticing prospects. For instance, in the case of the $\mathrm{LaAlO}_{3} / \mathrm{SrTiO}_{3}$ system, the internal field may be manipulated by directly writing charges on the surface [41,42]. Similar approaches may be used to modulate magnetism [43] or ferroelectricity [44] in other systems, thus significantly expanding the scopes of material design at perovskite interfaces. We conclude, therefore, that this kind of complex interplay may be used as a general tool to engineer potentially useful physical properties in atomically thin $\mathrm{ABO}_{3}$-perovskite structures.

This work was supported by the Spanish MAT201456063-C2-1-R, Severo Ochoa SEV-2015-0496 grant, the RyC-2012-11709 contract of J. G., and the Generalitat de Catalunya (2014 SGR 734) project. Electron microscopy observations at ORNL were supported by the U.S. Department of Energy (DOE), Basic Energy Sciences (BES), Materials Sciences and Engineering Division. M.S. acknowledges the support of MINECO through Grants No. MAT2016-77100-C2-2-P and No. FIS201348668-C2-2-P and of Generalitat de Catalunya (Grant No. 2014 SGR301). Calculations were performed at the Supercomputing Center of Galicia (CESGA). The authors are also grateful to Nico Dix for his collaboration in the growth of the films. Research at UCM sponsored by Fundación BBVA and Spanish MINECO MAT201566888-C3-3-R.

[1] R. E. Cohen, Nature (London) 358, 136 (1992).

[2] K. M. Rabe, C. H. Ahn, and J. M. -M. Triscone, Physics of Ferroelectrics: A Modern Prespective (Springer, Berlin, 2007).

[3] U. Aschauer and N. A. Spaldin, J. Phys. Condens. Matter 26, 122203 (2014).

[4] J. M. Rondinelli and C. J. Fennie, Adv. Mater. 24, 1961 (2012).

[5] T. H. Kim, D. Puggioni, Y. Yuan, L. Xie, H. Zhou, N. Campbell, P. J. Ryan, Y. Choi, J.-W. Kim, J. R. Patzner, S. Ryu, J. P. Podkaminer, J. Irwin, Y. Ma, C. J. Fennie, M. S. Rzchowski, X. Q. Pan, V. Gopalan, J. M. Rondinelli, and C. B. Eom, Nature (London) 533, 68 (2016).

[6] P. Aguado-Puente, P. Garcia-Fernández, and J. Junquera, Phys. Rev. Lett. 107, 217601 (2011).

[7] A. A. Balandin and D. L. Nika, Mater. Today 15, 266 (2012)

[8] M. Maldovan, Nature (London) 503, 209 (2013).
[9] N. Li, J. Ren, L. Wang, G. Zhang, P. Hänggi, and B. Li, Rev. Mod. Phys. 84, 1045 (2012).

[10] J. Hone, B. Batlogg, Z. Benes, A. T. Johnson, and J. E. Fischer, Science 289, 1730 (2000).

[11] G. Granger, D. Taubert, C. E. Young, L. Gaudreau, A. Kam, S. A. Studenikin, P. Zawadzki, D. Harbusch, D. Schuh, W. Wegscheider, Z. R. Wasilewski, A. A. Clerk, S. Ludwig, and A. S. Sachrajda, Nat. Phys. 8, 522 (2012).

[12] J. Junquera and P. Ghosez, Nature (London) 422, 506 (2003).

[13] C. Lichtensteiger, P. Zubko, M. Stengel, P. Aguado-Puente, J.-M. Triscone, P. Ghosez, and J. Junquera, in Oxide Ultrathin Film (Wiley-VCH, Berlin, 2011), pp. 230-265.

[14] M. Imada, A. Fujimori, and Y. Tokura, Rev. Mod. Phys. 70, 1039 (1998).

[15] D. G. Schlom and J. Mannhart, Nat. Mater. 10, 168 (2011).

[16] A. Ohtomo and H. Y. Hwang, Nature (London) 427, 423 (2004).

[17] S. Thiel, G. Hammerl, A. Schmehl, C. W. Schneider, and J. Mannhart, Science 313, 1942 (2006).

[18] N. Reyren, S. Thiel, A. D. Caviglia, L. F. Kourkoutis, G. Hammerl, C. Richter, C. W. Schneider, T. Kopp, A.-S. Ruetschi, D. Jaccard, M. Gabby, D. A. Muller, J.-M. Triscone, and J. Mannhart, Science 317, 1196 (2007).

[19] N. Nakagawa, H. Y. Hwang, and D. A. Muller, Nat. Mater. 5, 204 (2006).

[20] R. Pentcheva and W. E. Pickett, Phys. Rev. Lett. 102, 107602 (2009).

[21] M. Stengel, Phys. Rev. Lett. 106, 136803 (2011).

[22] See Supplemental Material at http://link.aps.org/ supplemental/10.1103/PhysRevLett.119.106102 for experimental and computational details, as well as additional STEM-EELS data and calculations, which includes Refs. [23-32].

[23] G. Herranz, F. Sánchez, N. Dix, M. Scigaj, and J. Fontcuberta, Sci. Rep. 2, 758 (2012).

[24] P. D. Nellist and S. J. Pennycook, Ultramicroscopy 78, 111 (1999).

[25] Y. K. E. Okunishi, I. Ishikawa, H. Sawada, F. Hosokawa, and M. Hori, Microsc. Microanal. 15, 164 (2009).

[26] R. Ishikawa, E. Okunishi, H. Sawada, Y. Kondo, F. Hosokawa, and E. Abe, Nat. Mater. 10, 278 (2011).

[27] R. Ishikawa, A. R. Lupini, F. Oba, S. D. Findlay, N. Shibata, T. Taniguchi, K. Watanabe, H. Hayashi, T. Sakai, I. Tanaka, Y. Ikuhara, and S. J. Pennycook, Sci. Rep. 4, 3778 (2014).

[28] P. R. Willmott, S. A. Pauli, R. Herger, C. M. Schlepütz, D. Martoccia, B. D. Patterson, B. Delley, R. Clarke, D. Kumah, C. Cionca, and Y. Yacoby, Phys. Rev. Lett. 99, 155502 (2007).

[29] P. E. Blöchl, Phys. Rev. B 50, 17953 (1994).

[30] M. Stengel and N. A. Spaldin, Nature (London) 443, 679 (2006).

[31] M. Stengel, N. A. Spaldin, and D. Vanderbilt, Nat. Phys. 5, 304 (2009).

[32] P. Bouvier and J. Kreisel, J. Phys. Condens. Matter 14, 3981 (2002).

[33] C. L. Jia, S. B. Mi, M. Faley, U. Poppe, J. Schubert, and K. Urban, Phys. Rev. B 79, 081405 (2009).

[34] C. Cantoni, J. Gazquez, F. Miletto Granozio, M. P. Oxley, M. Varela, A. R. Lupini, S. J. Pennycook, C. Aruta, U. S. di 
Uccio, P. Perna, and D. Maccariello, Adv. Mater. 24, 3952 (2012).

[35] A. J. Hatt and N. a. Spaldin, Phys. Rev. B 82, 195402 (2010).

[36] D. Vanderbilt and W. Zhong, Ferroelectrics 206, 181 (1998).

[37] C. Cazorla and M. Stengel, Phys. Rev. B 92, 214108 (2015).

[38] M. Stengel and J. Íniguez, Phys. Rev. B 92, 235148 (2015).

[39] N. A. Benedek and C. J. Fennie, J. Phys. Chem. C 117, 13339 (2013).

[40] J. Hong and D. Vanderbilt, Phys. Rev. B 87, 0064104 (2013).
[41] C. Cen, S. Thiel, G. Hammerl, C. W. Schneider, K. E. Andersen, C. S. Hellberg, J. Mannhart, and J. Levy, Nat. Mater. 7, 298 (2008).

[42] Y. Xie, C. Bell, Y. Hikita, S. Harashima, and H. Y. Hwang, Adv. Mater. 25, 4735 (2013).

[43] Z. Liao, M. Huijben, Z. Zhong, N. Gauquelin, S. Macke, R. J. Green, S. Van Aert, J. Verbeeck, G. Van Tendeloo, K. Held, G. A. Sawatzky, G. Koster, and G. Rijnders, Nat. Mater. 15, 425 (2016).

[44] H. N. Lee, H. M. Christen, M. F. Chisholm, C. M. Rouleau, and D. H. Lowndes, Nature (London) 433, 395 (2005). 